\title{
The Thinnest, Steepest, and Maximum Elevation Corneal Locations in Noncontact and Contact Lens Wearers in Keratoconus
}

\author{
Miguel Romero-Jiménez, OD, *† Jacinto Santodomingo-Rubido, PhD, $\neq$ \\ and Jose-Manuel González-Méijome, PhD†
}

Purpose: To assess the relationship between the thinnest corneal location and the steepest and maximum elevation corneal locations in subjects with keratoconus and the effect of gas permeable contact lens wear on the location of these points.

Methods: Sixty-one consecutive subjects (98 eyes) with keratoconus. Thirty-one (49 eyes) and 30 (49 eyes) subjects were gas permeable contact lens (CL-W) and non-contact lens wearers (N-CL), respectively. Thinnest, steepest, and maximum elevation corneal locations were evaluated from topographies collected with Pentacam Eye Scanner.

Results: In the entire sample and in N-CL and CL-W, the thinnest location does not overlap with the steepest or maximum elevation corneal locations (all $P>0.05$ ). The thinnest and maximum tangential curvature locations were found to be located further away from the geometric center of the cornea in CL-W versus $\mathrm{N}-\mathrm{CL}$ $(P<0.05)$.

Conclusion: The thinnest corneal location does not overlap with maximum axial and tangential curvatures or with the front and back elevation locations in keratoconus subjects. Contact lens wear does not affect this lack of overlapping.

Key Words: cornea, keratoconus, contact lenses

(Cornea 2012;0:1-6)

$\mathrm{K}^{\mathrm{s}}$ eratoconus is a progressive corneal disorder with an incidence of 1 per 2000 in the general population. ${ }^{1,2}$ It is a bilateral and asymmetric corneal degeneration characterized by localized corneal thinning, which leads to protrusion of the thinned cornea. Corneal thinning normally occurs in

Received for publication November 6, 2011; revision received March 8, 2012; accepted April 8, 2012.

From the *MGR Doctores, Madrid, Spain; $\uparrow$ Clinical \& Experimental Optometry Research Lab, Center of Physics, University of Minho, Braga, Portugal; and $\$$ Menicon Co., Ltd (Madrid Office), Majadahonda, Madrid, Spain.

The authors state that they have no proprietary interest in the products named in this article.

J. Santodomingo-Rubido is a full-time employee of Menicon Co., Ltd. M. Romero-Jiménez is undertaking a $\mathrm{PhD}$ partly funded by Menicon Co., Ltd., at the Clinical \& Experimental Optometry Research Lab, Center of Physics, University of Minho, Braga, Portugal.

Reprints: Miguel Romero-Jiménez, MGR Doctores, Vallehermoso St, 32 Bajo A, 28015, Madrid, Spain (e-mail: mrjlentes@gmail.com).

Copyright $(2012$ by Lippincott Williams \& Wilkins the inferior-temporal and the central cornea. Corneal protrusion causes high myopia and irregular astigmatism, affecting visual quality. Currently, a combination of corneal topographic findings (ie, inferior corneal steepening and superior-inferior corneal curvature asymmetry) and clinical signs (ie, Fleischer ring, Vogt striae, and corneal scarring) are commonly used to diagnose and monitor the disease. ${ }^{3,4}$ Although the etiology of keratoconus remains unclear, it is suspected to develop as a combination of genetic and environmental factors. ${ }^{5}$

To date, gas permeable contact lenses (CLs) represent the most common and successful treatment option for early to moderate cases of keratoconus because these lenses can correct relatively high levels of irregular astigmatism and thus substantially improve visual acuity. When CLs cannot be successfully fitted, most keratoconus subjects undertake penetrating keratoplasty surgery. In fact, it has been estimated that around $12 \%$ of keratoconus patients eventually require penetrating keratoplasty. ${ }^{6}$ However, in the last decade, new surgical procedures, including corneal ring segment implantation $^{7,8}$ and collagen cross-linking, ${ }^{9,10}$ have been developed to treat keratoconus.

Although previously studies have assessed the location of the steepest and thinnest corneal locations in keratoconus subjects, ${ }^{11,12}$ no previous studies have investigated in detail whether the thinnest corneal location overlaps with steepest and maximum elevation corneal locations in keratoconus. Additionally, previous studies have been undertaken using Placido-based corneal topography instruments. Currently, sophisticated instruments based on the Scheimpflug principle allow precise 3-dimensional reconstruction of the entire cornea shape ${ }^{13-17}$ Furthermore, it is not known whether CL wear affects these corneal locations. Therefore, the purpose of this study was to assess whether the thinnest corneal location overlaps with the steepest and maximum elevation corneal locations in keratoconus subjects and whether CL wear affects these corneal locations.

\section{MATERIALS AND METHODS}

\section{Patient Selection}

Ninety-eight eyes from 61 consecutive subjects were included in the study: 49 eyes from 31 subjects who have been wearing gas permeable CLs for a minimum period of 6 months (mean 27.5 months, $\mathrm{SD} \pm 4.3$ ) and 49 eyes from 30 subjects who had never worn CLs. All subjects included in the study 
were diagnosed with keratoconus according to the Keratoconus Severity Score. ${ }^{3}$ Eyes with a previous history of acute corneal hydrops or corneal surgery were excluded from the study. All subjects provided written informed consent to participate in the study. The study followed the Tenets of the Declaration of Helsinki and was approved by the Institutional Ethical Committee Review Board of MGR Doctores Ophthalmology Clinic.

\section{Data Collection}

Corneal topographies were taken and evaluated using the Pentacam Eye Scanner (Software version 1.16.r:23; Oculus, Inc, Wetzlar, Germany). Anterior segment reconstructions were produced with 25 single captures. On completion of every scan, the Pentacam software analyzed 12,500 points from the front and back corneal surfaces to generate a 3-dimensional image of the anterior segment.

The thinnest corneal location is automatically calculated by the instrument and represented in terms of $x$ and $y$ coordinates. To evaluate the steepest corneal location, axial and tangential front curvature maps were used. The maximum corneal elevation location was recorded from front and back elevation best-fit sphere topography maps. To determine the $x$ and $y$ coordinates of the steepest corneal location in the axial and tangential curvature maps and the highest location in maximum front and back elevation maps, 3 consecutive measurements were recorded manually and a mean was calculated. Additionally, minimum corneal thickness, maximum axial and tangential curvature powers, maximum front and back heights, mean central keratometry, corneal thickness at the maximum tangential curvature location, corneal volume, anterior chamber depth, and anterior chamber volume were also evaluated to ascertain whether CL wear affects the entire or just the anterior cornea.

Axial and tangential curvature front maps were evaluated using the American style map display setting with a relative scale of 61 colors and 1-diopter steps. Front and back elevation maps were also evaluated using the American style map display setting with a relative scale of 61 colors and a sphere reference shape with float and auto diameter options and $2.5 \mu \mathrm{m}$ steps. All $x$ and $y$ coordinates were transformed into vectors and angles to carry out the statistical analysis and to describe the distance and orientation of the different locations with regard to the instrument's measurement axis [ie, the point where the topography map shows the $(0,0)$ coordinate].

Superior and inferior and nasal and temporal corneal locations in right eyes were given positive and negative values, respectively, in relation to the instrument's measurement axis. Cones from left eyes have been horizontally inverted so that all cones are represented as if for the right eye.

In CL wearers, corneal topographies were taken immediately after lenses were removed. All measurements were performed by the same investigator (M.R.-J.).

\section{Statistical Analyses}

The mean and standard deviations were calculated for each vector and angle. Differences in each parameter were assessed separately. Two locations were considered different if statistically significant differences were found in vector, angle, or both of them. Paired sample $t$ tests were used to assess differences in vectors and angles between the different variables measured for the entire sample group and in non-contact (N-CL) and contact lens wearers (CL-W). Independent sample $t$ tests were used to assess differences in vectors and angles between N-CL and CL-W. Pearson product-moment correlations were used to assess relationships between the distance to the instrument's measurement axis and the distances from the thinnest to the other reference points. Statistical analyses were performed using SPSS 15.0 software (SPSS, Inc, Chicago, IL). The level of statistical significance was taken as $5 \%$.

\section{RESULTS}

\section{Subjects' Demographics}

The entire sample consisted of 98 eyes (49 N-CL and 49 CL-W) of 61 subjects, 42 of which were men (69\%). Thirty-two and 29 subjects were CL-W and N-CL, respectively. The mean age $( \pm \mathrm{SD})$ for the entire sample was $35.4 \pm 10.0$ (range, 12.2$67.5)$ years. The mean ages $( \pm \mathrm{SD})$ in $\mathrm{N}-\mathrm{CL}$ and $\mathrm{CL}-\mathrm{W}$ were $35.8 \pm 9.1$ (range, 22.3-65.1) years and 35.0 \pm 11.9 (range, $12.2-67.5)$ years, respectively $(P=0.758)$.

\section{Entire Sample}

The thinnest corneal location was found to be located in a different point of the cornea in comparison with both the maximum curvature (axial and tangential) and the maximum elevation (front a back) corneal locations (Fig. 1; Table 1).

A strong correlation was found between the distance from the vertex normal and (1) the distance between the thinnest and the axial $(r=0.963, P<0.001)$, (2) the thinnest and the tangential $(r=0.937, P<0.001),(3)$ the thinnest and the front $(r=0.881, P<0.001)$, and (4) the thinnest and the back elevation $(r=0.855, P<0.001)$ corneal locations.

\section{Effects of CL Wear}

The thinnest corneal location did not overlap with maximum curvature (axial and tangential) and maximum elevation (front and back) corneal locations in the N-CL and CL-W groups, when assessed separately (all $P>0.05$; Tables 2 and 3 ).

When comparing N-CL versus CL-W, the locations of the thinnest, maximum axial curvature, and maximum elevation (front and back) locations were found to be similar between groups (all $P>0.05$ ), but the maximum tangential curvature locations were found to be different between these 2 groups $(P<0.05)$. However, CL-W showed the thinnest, maximum tangential curvature, and maximum front elevation locations to be positioned further away from the instrument's measurement axis in comparison to N-CL (Figs. 2, 3).

The thickness at the thinnest corneal location, mean central corneal power, and maximum front and back elevation heights between N-CL and CL-W were found to be similar (all $P>0.05$ ). However, significant differences were found in the maximum axial and tangential curvatures between N-CL and CL-W (both $P<0.05$; Table 4). 


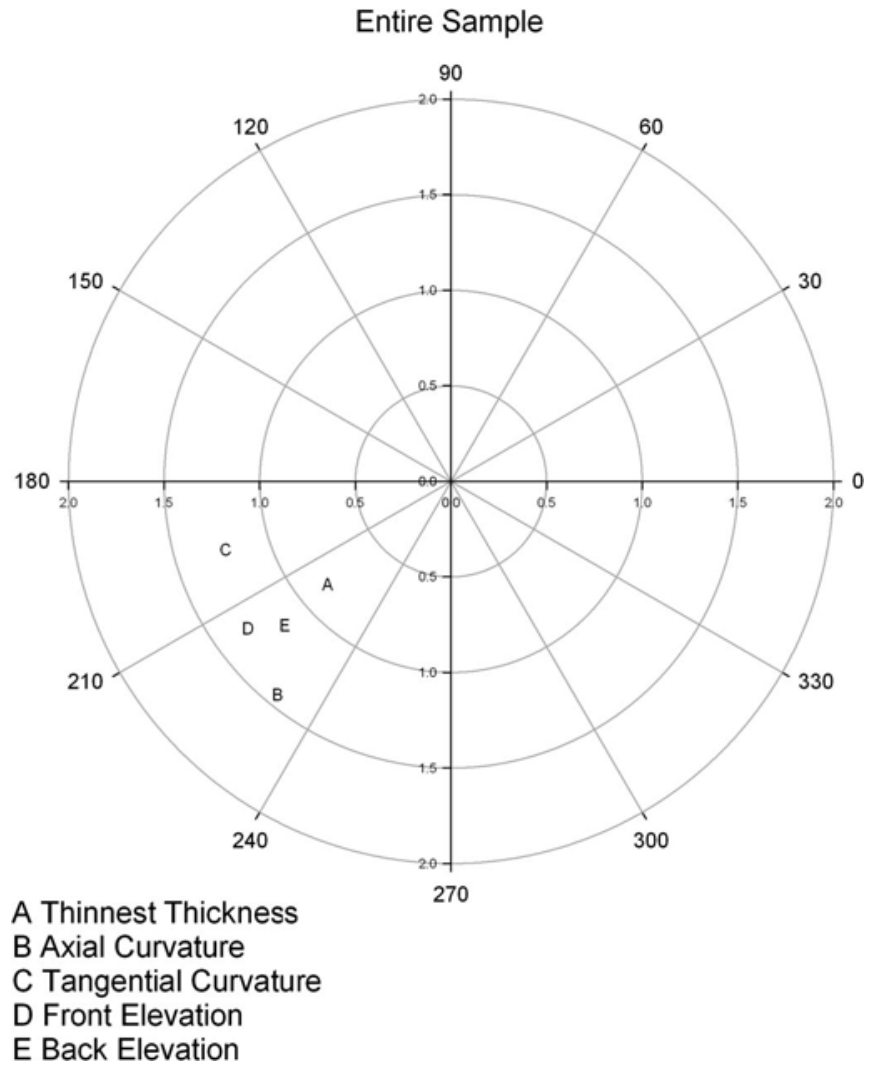

FIGURE 1. Position (angle and distance) of the reference points for the entire sample with regard to the instrument's measurement axis. Angles and distances are expressed in degrees and millimeters, respectively.

No significant differences were found in the thickness at the maximum tangential curvature location, corneal volume, anterior chamber volume, or the anterior chamber depth between N-CL and CL-W (all $P>0.05$; Table 5).

TABLE 1. Comparison Between the Thinnest Location and Maximum Curvature and Elevation Locations

\begin{tabular}{|c|c|c|c|c|c|c|}
\hline \multicolumn{7}{|c|}{ Vectors } \\
\hline \multicolumn{2}{|c|}{ Coordinate 1 (C1) } & \multicolumn{3}{|c|}{ Coordinate 2 (C2) } & $\mathrm{C} 1-\mathrm{C} 2 *$ & $\boldsymbol{P}$ \\
\hline \multirow[t]{4}{*}{ Thinnest } & \multirow[t]{4}{*}{$0.84( \pm 0.29)$} & Axial & \multicolumn{2}{|c|}{$1.44( \pm 0.96)$} & 0.62 & 0.001 \\
\hline & & Tangential & \multicolumn{2}{|c|}{$1.23( \pm 0.71)$} & 0.57 & 0.001 \\
\hline & & Front elevation & \multicolumn{2}{|c|}{$1.31( \pm 0.51)$} & 0.47 & 0.001 \\
\hline & & Back elevation & \multicolumn{2}{|c|}{$1.15( \pm 0.41)$} & 0.31 & 0.030 \\
\hline \multicolumn{7}{|c|}{ Angles } \\
\hline \multicolumn{2}{|c|}{ Coordinate 1} & \multicolumn{4}{|c|}{ Coordinate 2} & $P$ \\
\hline \multirow[t]{4}{*}{ Thinnest } & \multirow[t]{4}{*}{$220^{\circ}( \pm 32)$} & Axial & & \multicolumn{2}{|c|}{$231^{\circ}( \pm 70)$} & 0.127 \\
\hline & & \multicolumn{2}{|l|}{ Tangential } & \multicolumn{2}{|c|}{$197^{\circ}( \pm 54)$} & 0.001 \\
\hline & & \multicolumn{2}{|c|}{ Front elevation } & \multicolumn{2}{|c|}{$216^{\circ}( \pm 29)$} & 0.389 \\
\hline & & \multicolumn{2}{|c|}{ Back elevation } & \multicolumn{2}{|c|}{$221^{\circ}( \pm 28)$} & 0.855 \\
\hline
\end{tabular}

TABLE 2. Comparison Between the Thinnest Location and Maximum Curvature and Elevation Locations for $\mathrm{N}-\mathrm{CL}$

\begin{tabular}{|c|c|c|c|c|c|c|}
\hline \multicolumn{7}{|c|}{ Vectors } \\
\hline \multicolumn{2}{|c|}{ Coordinate $1(\mathrm{C} 1)$} & \multicolumn{3}{|c|}{ Coordinate 2 (C2) } & C1-C2* & $P$ \\
\hline \multirow[t]{4}{*}{ Thinnest } & \multirow[t]{4}{*}{$0.76( \pm 0.30)$} & Axial & \multicolumn{2}{|c|}{$1.22( \pm 0.96)$} & 0.69 & 0.001 \\
\hline & & Tangential & \multicolumn{2}{|c|}{$0.93( \pm 0.71)$} & 0.54 & 0.034 \\
\hline & & Front elevation & \multicolumn{2}{|c|}{$1.14( \pm 0.51)$} & 0.42 & 0.001 \\
\hline & & Back elevation & \multicolumn{2}{|c|}{$1.04( \pm 0.41)$} & 0.31 & 0.001 \\
\hline \multicolumn{7}{|c|}{ Angles } \\
\hline \multicolumn{2}{|c|}{ Coordinate 1} & \multicolumn{4}{|c|}{ Coordinate 2} & $P$ \\
\hline \multirow[t]{4}{*}{ Thinnest } & \multirow[t]{4}{*}{$201^{\circ}( \pm 38)$} & \multicolumn{2}{|l|}{ Axial } & \multicolumn{2}{|c|}{$269^{\circ}( \pm 63)$} & 0.041 \\
\hline & & \multicolumn{2}{|l|}{ Tangential } & \multicolumn{2}{|c|}{$264^{\circ}( \pm 56)$} & 0.002 \\
\hline & & \multicolumn{2}{|c|}{ Front elevation } & \multicolumn{2}{|c|}{$227^{\circ}( \pm 23)$} & 0.801 \\
\hline & & \multicolumn{2}{|c|}{ Back elevation } & \multicolumn{2}{|c|}{$2214^{\circ}( \pm 23)$} & 0.952 \\
\hline
\end{tabular}

Vectors are expressed in millimeters.

*Vectorial difference between the thinnest location and the maximum curvature and elevation locations.

\section{DISCUSSION}

It has been previously reported that the Pentacam instrument provides high sensitivity and specificity in keratoconus detection ${ }^{13,14}$ and high reproducibility and repeatability in measuring corneal thickness, ${ }^{15}$ power ${ }^{16}$ and posterior elevation. ${ }^{17}$ Therefore, the device used in this study is well suited for assessing corneal shape in keratoconus subjects.

\section{Entire Sample}

The thinnest corneal location did not overlap with the maximum axial and tangential curvature or with the maximum front and back elevation corneal locations in this study. The latter is in agreement with previous reports in normal ${ }^{18}$ and keratoconus subjects. ${ }^{11,12}$

TABLE 3. Comparison Between the Thinnest Location and Maximum Curvature and Elevation Locations for CL-W

\begin{tabular}{|c|c|c|c|c|c|c|}
\hline \multicolumn{7}{|c|}{ Vectors } \\
\hline \multicolumn{2}{|c|}{ Coordinate 1 (C1) } & \multicolumn{3}{|c|}{ Coordinate 2 (C2) } & $\mathrm{C} 1-\mathrm{C}_{2}$ * & $P$ \\
\hline \multirow[t]{4}{*}{ Thinnest } & \multirow[t]{4}{*}{$0.82( \pm 0.29)$} & Axial & \multicolumn{2}{|c|}{$1.21( \pm 0.96)$} & 0.61 & 0.001 \\
\hline & & Tangential & \multicolumn{2}{|c|}{$1.33( \pm 0.71)$} & 0.59 & 0.001 \\
\hline & & Front elevation & \multicolumn{2}{|c|}{$1.23( \pm 0.51)$} & 0.53 & 0.001 \\
\hline & & Back elevation & \multicolumn{2}{|c|}{$1.09( \pm 0.41)$} & 0.31 & 0.001 \\
\hline \multicolumn{7}{|c|}{ Angles } \\
\hline \multicolumn{2}{|c|}{ Coordinate 1} & \multicolumn{4}{|c|}{ Coordinate 2} & $P$ \\
\hline \multirow[t]{4}{*}{ Thinnest } & \multirow[t]{4}{*}{$233^{\circ}( \pm 24)$} & Axial & & \multicolumn{2}{|c|}{$267^{\circ}( \pm 76)$} & 0.829 \\
\hline & & \multicolumn{2}{|l|}{ Tangential } & \multicolumn{2}{|c|}{$266^{\circ}( \pm 48)$} & 0.089 \\
\hline & & \multicolumn{2}{|c|}{ Front elevation } & \multicolumn{2}{|c|}{$220^{\circ}( \pm 33)$} & 0.285 \\
\hline & & \multicolumn{2}{|c|}{ Back elevation } & \multicolumn{2}{|c|}{$236^{\circ}( \pm 32)$} & 0.830 \\
\hline
\end{tabular}

Vectors are expressed in millimeters.

*Vectorial difference between the thinnest location and the maximum curvature and elevation locations. 


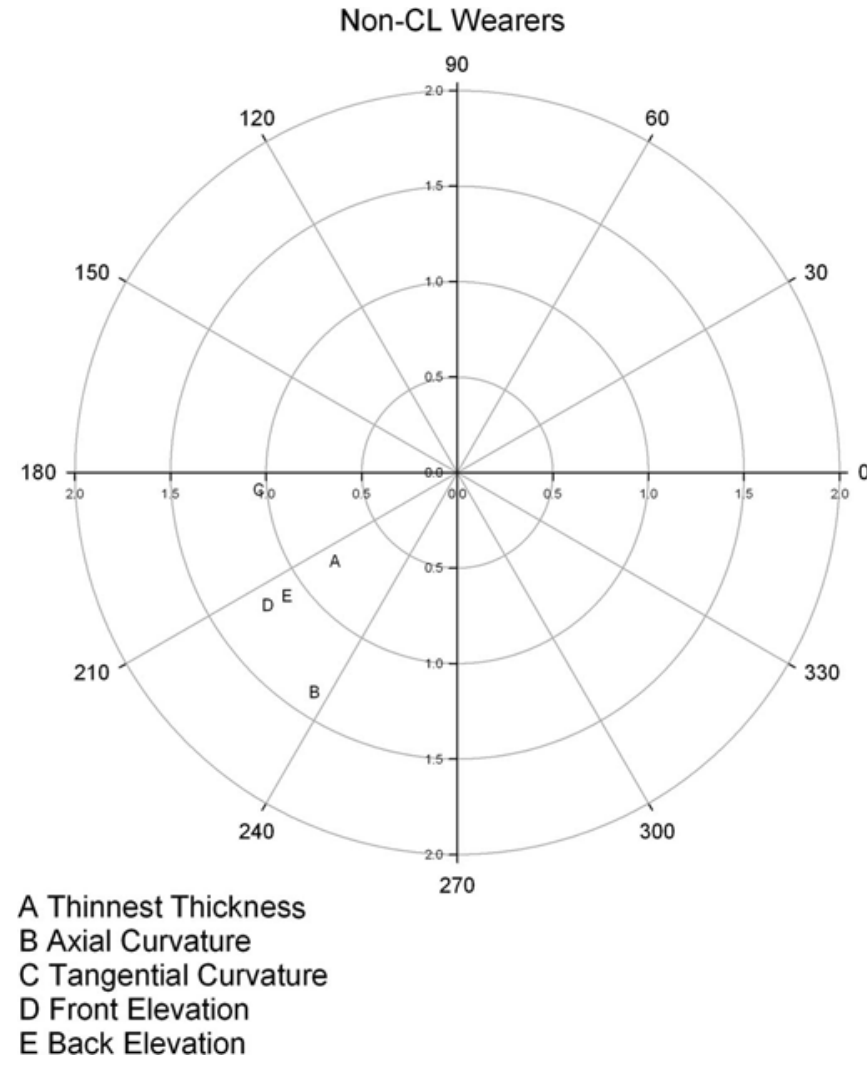

FIGURE 2. Position (angle and distance) of the reference points in N-CL with regard to the instrument's measurement axis. Angles and distances are expressed in degrees and millimeters, respectively.

We found that the greater the distance between the thinnest location and the other reference points (ie, axial, tangential, front, and back elevations), the greater the distance between the reference points and the normal corneal vertex and vice versa. The latter suggests that the reference locations are closer and further away from each other in central and peripheral cones, respectively.

\section{Effects of CL Wear}

The lack of overlapping between the thinnest and the maximum curvature (axial and tangential) and maximum elevation (front and back) locations found in the entire sample and in both N-CL and CL-W suggests that this is likely to be an anatomical feature of keratoconus disease and that $\mathrm{CL}$ wear does not affect this lack of overlapping.

Some differences, however, were found between N-CL and CL-W in our study. The thinnest and maximum tangential curvature locations were found to be positioned slightly further away from the instrument's measurement axis in CL-W in comparison to N-CL. Additionally, a 3.00 diopter difference was found in the maximum tangential curvature power between N-CL and CL-W. It has been previously reported that gas permeable CLs can flatten the central cornea and more particularly the corneal epithelium, ultimately leading to a potential displacement of the cone apex. ${ }^{19,20}$ Therefore,

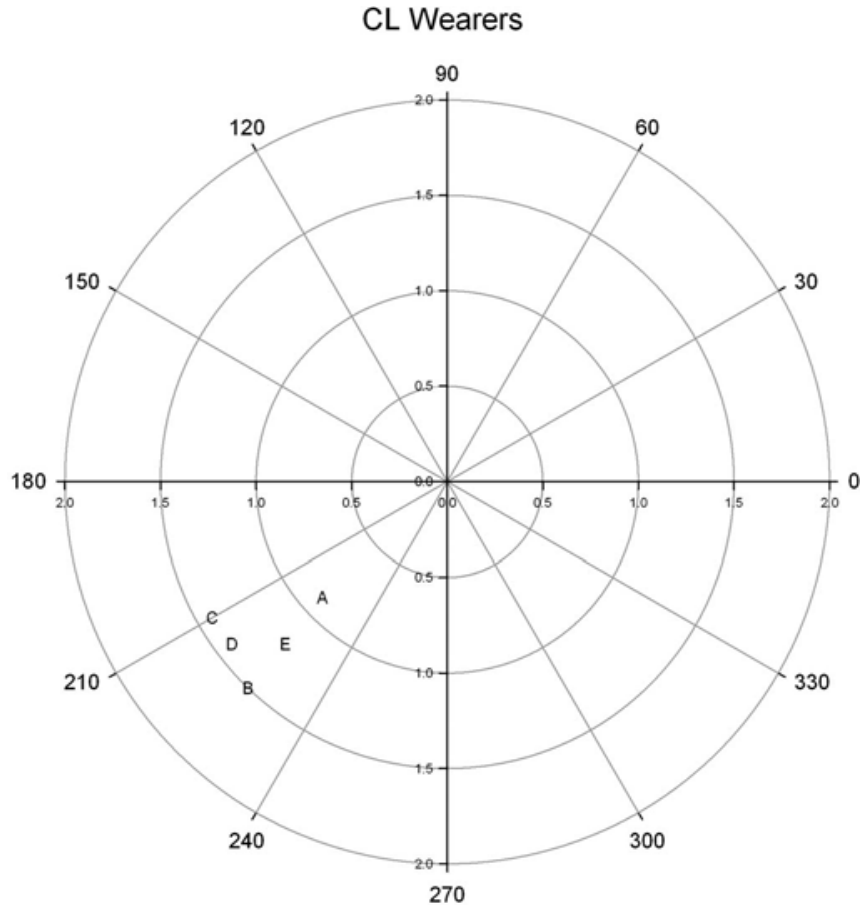

A Thinnest Thickness

B Axial Curvature

C Tangential Curvature

D Front Elevation

E Back Elevation

FIGURE 3. Position (angle and distance) of the reference points in CL-W with regard to the instrument's measurement axis. Angles and distances are expressed in degrees and millimeters, respectively.

it is possible that the physical pressure exerted by the CL over the keratoconic cornea modifies the anterior corneal curvature and power and perhaps the thinnest corneal location as well. The latter is supported by a previous study, which reported that keratoconus subjects fitted with gas permeable CLs showed greater corneal irregularity and higher central curvature power than N-CL keratoconus subjects. ${ }^{21}$ It is also known

TABLE 4. Thinnest Corneal Thickness, Mean Central Keratometry, Maximum Axial and Tangential Curvatures, and Maximum Front and Back Elevation Values in N-CL and CL-W

\begin{tabular}{lrrrrrr}
\hline & \multicolumn{4}{c}{ Value } \\
\cline { 2 - 3 } \multicolumn{1}{c}{ Measurement } & \multicolumn{1}{c}{ N-CL (n= 49) } & & \multicolumn{1}{c}{ CL-W (n= 49) } & \\
\cline { 2 - 3 } & Mean & \pm SD & & Mean & \pm SD & $\boldsymbol{P}$ \\
\hline Thickness & 450.73 & 51.91 & 454.86 & 51.82 & 0.695 \\
CK & 49.38 & 5.46 & 48.64 & 3.55 & 0.430 \\
Max keratometry, axial & 56.71 & 8.00 & 53.68 & 5.16 & 0.028 \\
Max keratometry, tang & 57.95 & 7.74 & 55.14 & 5.81 & 0.045 \\
Max elev, front & 39.29 & 22.10 & 31.33 & 21.22 & 0.072 \\
Max elev, back & 65.37 & 30.45 & 62.29 & 35.49 & 0.646 \\
\hline
\end{tabular}

Curvature is expressed in diopters. Thickness and elevations are expressed in microns.

CK, central keratometry; Elev, elevation; Max, maximum; Tang, tangential. 
TABLE 5. Thinnest Thickness at the Maximum Tangential Curvature Point, Corneal Volume, and Anterior Chamber Volume and Depth

\begin{tabular}{lrrrrrr}
\hline & \multicolumn{2}{c}{$\mathbf{N - C L}(\mathbf{n}=\mathbf{4 9})$} & & \multicolumn{2}{c}{ CL-W $(\mathbf{n}=\mathbf{4 9})$} & \\
\cline { 2 - 3 } \multicolumn{1}{c}{ Measurement } & Mean & \pm SD & & Mean & \pm SD & $\boldsymbol{P}$ \\
\hline Tangential thickness & 464.12 & 51.44 & & 483.53 & 51.08 & 0.327 \\
CV & 57.66 & 3.84 & & 59.16 & 4.75 & 0.090 \\
ACV & 199.57 & 35.84 & & 187.35 & 35.03 & 0.091 \\
ACD & 3.41 & 0.352 & & 3.34 & 0.348 & 0.064 \\
\hline
\end{tabular}

All values are expressed in microns.

$\mathrm{ACD}$, anterior chamber depth; $\mathrm{ACV}$, anterior chamber volume; $\mathrm{CV}$, corneal volume.

that the cone apex gets displaced toward the corneal periphery with the normal progression of the disease, and higher central keratometric powers are commonly associated with more advanced stages of keratoconus disease. ${ }^{1,2}$ In our study, N-CL and CL-W were well matched in terms of age, corneal thickness, and central curvature power; however, it is unclear whether the further away corneal locations of the thinnest and maximum tangential found in CL-W in comparison to $\mathrm{N}-\mathrm{CL}$ in this study are attributed to CL wear or the normal evolution of the disease.

No significant differences in front and back elevations were found in this study between N-CL and CL-W, neither in location nor in height. It has been shown that front and back elevation maps provide a more accurate representation of the anterior corneal surface than curvature maps because they are independent of axis, orientation, and position. ${ }^{14}$ Furthermore, elevation maps have been reported to be useful in the diagnosis and monitoring of keratoconus disease. ${ }^{22}$ Although gas permeable CL wear is commonly associated with flattening of the anterior central cornea, the use of these lenses is unlikely to change the true shape of the cornea (ie, front and back corneal elevations). However, our results are in contrast with a recent cross-sectional study that evaluated the effects of gas permeable CL wear in keratoconus subjects. ${ }^{23}$ After close to 2 years of follow-up, lower front and higher back corneal elevations, respectively, were found with $\mathrm{CL}$ wear in comparison to baseline but no significant changes were observed in corneal elevation or curvature in N-CL in comparison to baseline. $^{23}$ Although CL wearers showed reduced corneal thickness and increased central curvature power in comparison to N-CL after 2 years, the latter might be attributed to differences in disease severity between the groups in that study. $^{23}$

To assess the true effect of CL wear on the corneal shape of keratoconus, it would have been ideal to discontinue CL wear for a relatively long period (eg, 1 month) and then compare the results with those collected a few minutes after lens removal. However, the latter was not possible because keratoconus subjects rely on their CLs to achieve adequate visual function.

No statistical differences in corneal volume, anterior chamber depth, anterior chamber volume, and corneal thickness at the maximum tangential curvature corneal location were found between N-CL and CL-W. Although we were expecting a slight reduction in corneal thickness at the maximum tangential corneal location in CL-W in comparison to N-CL as a result of CL-induced corneal flattening, an increase in corneal thickness was found. Furthermore, we observed smaller anterior chamber depth and anterior chamber volume in CL-W versus N-CL. Despite the fact that keratoconus has been typically described in the literature as a noninflammatory disease, recent studies have shown that it is accompanied by an increase in proinflammatory cytokines in the tears in comparison to normal subjects, ${ }^{24}$ the inflammatory response being even greater with CL wear. ${ }^{25}$ It is, therefore, possible that the physical pressure induced by CL wear causes a local, slight, and superficial inflammation of the anterior cornea, leading to an increase in local thickness and volume.

In summary, the thinnest corneal location does not overlap with the steepest and maximum elevation corneal locations in keratoconus, and CL wear does not seem to affect this lack of overlapping. We suggest that CL wear in keratoconus could flatten the corneal apex, displacing the thinnest and maximum tangential curvature corneal locations. However, longitudinal studies are required to ascertain whether the change found in these corneal locations is attributed to CL wear per se or to the normal progression of the disease.

\section{REFERENCES}

1. Rabinowitz YS. Keratoconus. Surv Ophthalmol. 1998;42:297-319.

2. Romero-Jiménez M, Santodomingo-Rubido J, Wolffsohn J. Keratoconus: a review. Contact Lens Anterior Eye. 2010;33:157-166.

3. McMahon TT, Szczotka-Flynn L, Barr JT, et al. A new method for grading the severity of keratoconus: the keratoconus severity score (KSS). Cornea. 2006;25:794-799.

4. Perry HD, Buxton JN, Fine BS. Round and oval cones in keratoconus. Ophthalmology. 1980;87:905-909.

5. Edwards M, McGhee CN, Dean S. The genetics of keratoconus. Clin Experiment Ophthalmol. 2001;29:345-351.

6. Gordon MO, Steger-May K, Szczotka-Flynn L, et al; Clek Study Group. Baseline factors predictive of incident penetrating keratoplasty in keratoconus. Am J Ophthalmol. 2006;142:923-930.

7. Kyminois GD, Siganos CS, Tsikils NS, et al. Long-term follow-up of intacs in keratoconus. Am J Ophthalmol. 2007;143:236-244.

8. Colin J, Malet FJ. Intacs for the correction of keratoconus: two-year follow-up. J Cataract Refract Surg. 2007;33:69-74.

9. Wollensak G, Spoerl E, Seiler T. Riboflavin/ultraviolet-A-induced collagen crosslinking for the treatment of keratoconus. Am J Ophthalmol. 2003;135:620-627.

10. Caporossi A, Mazzotta C, Baiocchi S, et al. Long-term results of riboflavin ultraviolet a corneal collagen cross-linking for keratoconus in Italy: the Siena eye cross study. Am J Ophthalmol. 2010;149:585-593.

11. Demirbas NH, Pflugfelder SC. Topographic pattern and apex location of keratoconus on elevation topography maps. Cornea. 1998;17:476-484.

12. Auffarth GU, Wang L, Völcker HE. Keratoconus evaluation using the Orbscan Topography System. J Cataract Refract Surg. 2000;26: 222-228.

13. De Sanctis U, Loiacono C, Richiardi L, et al. Sensitivity and specificity of posterior corneal elevation measured by Pentacam in discriminating keratoconus/subclinical keratoconus. Ophthalmology. 2008;115:1534-1539.

14. Miháltz K, Kovács I, Takács A, et al. Evaluation of keratometric, pachymetric, and elevation parameters of keratoconic corneas with pentacam. Cornea. 2009;28:976-980.

15. De Sanctis U, Missolungi A, Mutani B, et al. Reproducibility and repeatability of central corneal thickness measurement in keratoconus using the rotating Scheimpflug camera and ultrasound pachymetry. Am J Ophthalmol. 2007;144:712-718. 
16. Kawamorita T, Uozato H, Kamiya K, et al. Repeatability, reproducibility, and agreement characteristics of rotating Scheimpflug photography and scanning-slit corneal topography for corneal power measurement. $J$ Cataract Refract Surg. 2009;35:127-133.

17. Chen D, Lam AK. Intrasession and intersession repeatability of the Pentacam system on posterior corneal assessment in the normal human eye $J$ Cataract Refract Surg. 2007;33:448-454.

18. Liu Z, Huang AJ, Pflugfelder SC. Evaluation of corneal thickness and topography in normal eyes using the Orbscan corneal topography system. Br J Ophthalmol. 1999;83:774-778.

19. Maeda N, Klyce SD, Hamamo H. Alteration of corneal asphericity in rigid gas permeable contact lens induced warpage. CLAO J. 1994;20:27-31.

20. Wilson SE, Lin DT, Klyce SD, et al. Topographic changes in contact lens induced corneal warpage. Ophthalmology. 1990;97:734-744.
21. Szcotka LB, Rabinowitz YS, Yang H. Influence of contact lens wear on the corneal topography of keratoconus. CLAO J. 1996;22:270-273.

22. Nilforoushan MR, Speaker M, Marmor M, et al. Comparative evaluation of refractive surgery candidates with Placido topography, Orbscan II, Pentacam, and wavefront analysis. J Cataract Refract Surg. 2008;34: 623-631.

23. Hwang JS, Lee JH, Wee WR, et al. Effects of multicurve RPG contact lens use on topographic changes in keratoconus. Korean J Ophthalmol. 2010;24:201-206.

24. Lema I, Durán JA. Inflammatory molecules in the tears of patients with keratoconus. Ophthalmology. 2005;112:654-659.

25. Lema I, Durán JA, Ruiz C, et al. Inflammatory response to contact lenses in patients with keratoconus compared with myopic subjects. Cornea. 2008;27:758-763. 